\title{
The unique national minority traditional patterns,texture and relevant cultural information research strategy of digital protection in Gansu
}

\author{
Gang Zheng $^{1, \mathrm{a}}$, Xiangwei $\mathrm{Li}^{2, \mathrm{~b}}$ and Jundi Wang ${ }^{2, \mathrm{c}}$ \\ Lanzhou Institute of Technology,Lanzhou Gansu,730050China; \\ a32028095@qq.com, b497064558@qq.com, ㄷ511378759@qq.com
}

Keywords: Gansu unique Minority;Traditional culture;Design Pattern;Information Digitization

\begin{abstract}
Unique ethnic minorities traditional patterns and texture in Gansu province and the related cultural relics item is with strong ethnic characteristics of national art, It is the crystallization of minority diligence and wisdom under the regional environment in Gansu. Study how digital media technology and Gansu Minority unique traditional motifs and patterns and other cultural resources of mutual integration, proposed Gansu minority traditional culture unique research strategy of protection and inheritance.
\end{abstract}

\section{Introduction}

Gansu is a multi-ethnic province.The province has 54 ethnic minorities, including the Hui, Tibetan, Dongxiang, Tu, Yugur, Bonan, Mongolian, Sarah ethnic Kazakh, Manchu and other 16 ethnic minorities living in Gansu for a long period of time, the Yugur, Dongxiang, Baoan for the Gansu region unique ethnic minorities, with the characteristics of Gansu regional culture. With the development of cultural heritage digitization process, changing the traditional way of information circulation and sharing.Gansu unique ethnic patterns and texture are hand-drawn form, has not yet combined with digital tools.It is very urgent and important to set up the database of the traditional patterns and texture of the ethnic minorities in Gansu and the digitalization of the cultural resources of the ethnic minorities in Gansu.

\section{Gansu unique conservation status of minority cultures}

Minorities in Yugu, Dongxiang and Baoan ethnic minorities in Gansu Province are faced with the rapid development of new media and lack of effective digital technology to protect, spread and inherit. specifically in:

\section{1 Cultural protection lags behind}

At present, the cultural studies of ethnic minorities in Gansu are relatively independent, single research model, lack of horizontal linkages and systematic research.Although many efforts have been made to protect the cultural relics, such as: making relevant cultural relics protection laws and regulations, determining minority cultural heritage, establishing minority cultural centers, and carrying out intangible cultural heritage declaration, etc ${ }^{[1]}$. But the lack of modern digital media technology integration.

\section{2 . 2 A single culture dissemination way}

The spread of unique ethnic minority cultural information and resources in Gansu province determines the degree of minority cultures understood and accepted.The spread of a single form restricts the development of minority cultures,Transmitted backward minority cultures face disappear or have been disappeared ${ }^{[2]}$. 


\section{2 . 3 Cultural inheritance form limitations}

Prerequisite for the development of minority culture is how to inherit.Gansu's unique cultural heritage of ethnic minorities is mainly used in oral communication and personal experience,This inheritance relationships conducted by the way, the lack of diversity and breadth of features in the form of heritage, development of cultural derivative products lack.

\section{3 . Digital protection of unique ethnic cultural resources in Gansu province}

\section{3 . 1 The research content and significance}

Gansu minority traditional pattern and the related culture unique item information digital protection, it is the traditional culture and the study of the combination of digital media technology.

3 . 1 . 1 Will be unique in Gansu minority patterns sexture and relevant cultural relics objects in the collection, summary, classification, integration.Combining art and digital media, the construction of Gansu minority traditional pattern and unique patterns information database.To promote study and application of Gansu minority traditional culture unique to a more systematic and systematic way.

3 . 1 . 2 Unique ethnic minorities in Gansu province in a lot of designs, patterns and related clothing, utensils, such as cultural relics.Therefore, for graphic patterns, pattern using two-dimensional vector data processing method for digital generation;For clothing, accessories, utensils and building local structure using 3d scanning, 3d simulation modeling and virtual reality interactive technology of digital generation.

3 . 1 . 3 Construction of Gansu unique minority traditional pattern and the pattern information database system.First, the system USES the information system based on pattern and pattern structure data dictionary ${ }^{[3]}$, overcome the problem of high redundancy traditional way;Secondly retrieval methods of the system mass pattern based on compressed domain retrieval techniques, greatly improve the real-time performance and the effectiveness of retrieval, reduces the computational complexity of data;Finally, to retrieve the user to quickly find and patterns related to cultural relics, and extract the related information, etc.

3 . 1 . 4 Research is aimed at inheriting and carrying forward, so how to better research results will be spread and make every minority nationality traditional culture lovers can quickly and efficiently to obtain relevant information is particularly important.Web-based virtual interaction is adopted to establish the network interactive digital protection platform of unique national minority material cultural heritage resources in Gansu province have the effect of integration, protection, communication and inheritance.

\section{3 . 2 The research method and train of thought}

First of all, the unique ethnic minority traditional pattern of Gansu province and a theoretical research related to cultural relics,Using the theory of network and library resources collection related pictures and data;Secondly, for the Sunan Yugur Autonomous County、Gansu Province Linxia Hui Autonomous Prefecture、Gansu province JiShiShan、Salar autonomous county and other places for field trips, Collection of Gansu unique minority traditional patterns and relevant cultural information, such as clothing, utensils, etc,Will be collected patterns and related artifacts generated digitally, through digital means to form minority culture digital information system;Finally, the digital media interaction design research,Familiar with related digital expression interaction design 
technology, The traditional patterns and relevant cultural information and interaction design practice and a series of methods for the combination of research,As shown in fig 1.

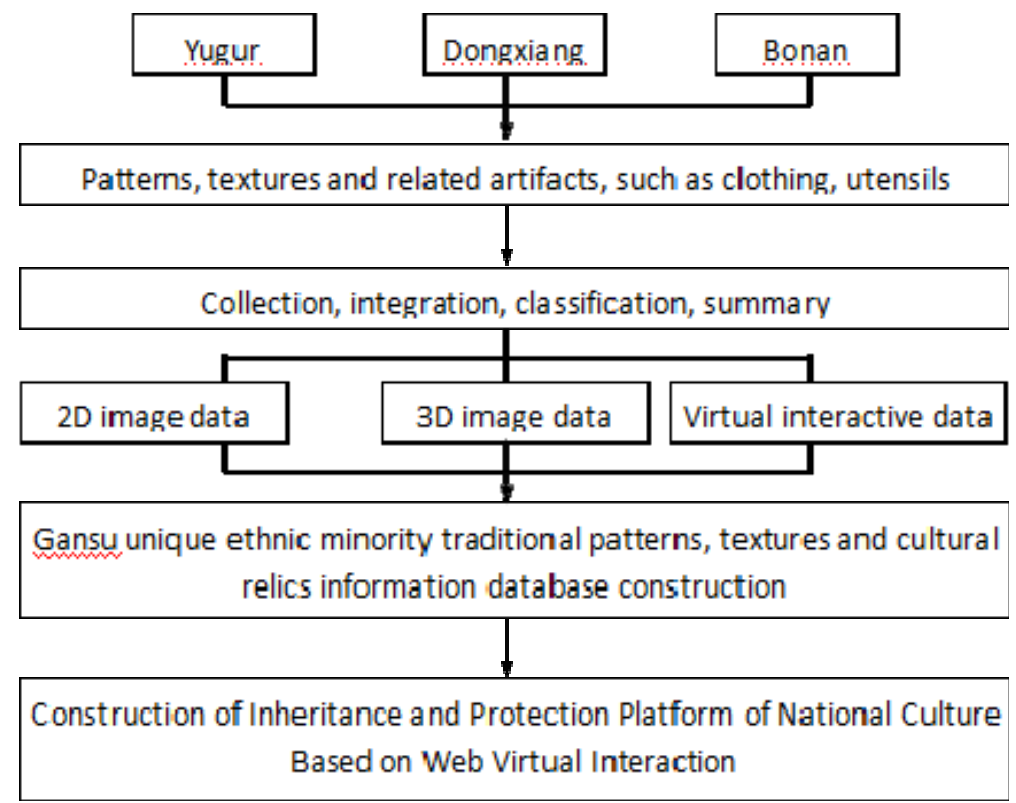

fig 1 The digitized construction overall frame

\section{Research strategy and the key technology}

Involve many ethnic minorities, Research is wide and Large amount of data collection.The research involves the Yugur, Dongxiang and Bonan.Minorities live relatively scattered, there are a lot of traditional pattern and cultural relics, need to spend a lot of time visiting investigation and data collection.Minority cultural center and cultural heritage is the key of the visit, the data collection mainly adopts shot with planar scanning, the video image acquisition and the method of combining the 3d scanning way.

Digital patterns and cultural relics items generated.Digital patterns and cultural relics refers to $2 \mathrm{~d}$ and $3 \mathrm{~d}$ digital image generation, Required for the process of the digital image data has high "fidelity",The digital data required in such aspects as form, texture, size, as far as possible consistent with the original data ${ }^{[4]}$,As far as possible keep minority culture national characteristic of the item.

Construction of Data Dictionary in Information Database System.Data dictionary refers to data items of data, data structure, data flow, data storage, processing logic, such as external entities are defined and described, its purpose is to each of the elements in the data flow diagram to make detailed instructions.Another difficulty is that in the study of how to integrate minority traditional pattern classification, based on the traditional pattern of feature extraction and classification, realize the pattern classification and computer logical mapping, on the basis of the traditional pattern of abstraction and coding, design related database model.

Based on the design of compressed domain feature extraction and retrieval technology.In this study, the formation of the system platform, the search is also a shortcut to test the system an important indicator.The system retrieval method adopts the feature extraction and retrieval technology of massive pattern based on compressed domain ${ }^{[5]}$. As shown in Fig2 and Fig3.In the process of realization, it is difficult to analyze the application situation of the traditional patterns 
involved in the static and dynamic image literatures, the way to extract the pattern image features and the frequency analysis of the patterns in the application process.
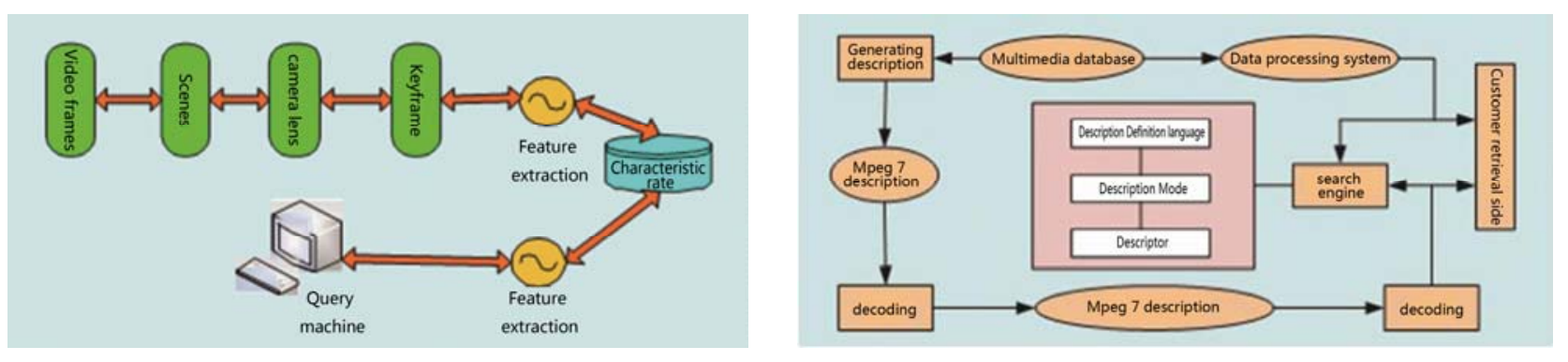

Fig2 Content-based video retrieval and mining structure diagram Fig3 Based on the MPEG video retrieval and mining system model

\section{5 . Summary}

Velocity anomaly rapid development today, information technology, the digital protection of cultural heritage have been can't just stay on the database as the platform of media, the future can also with the Internet, mobile Internet and new information technologies such as cloud computing and media, in order to more effectively protect, exhibition, repair and restoration of cultural heritage, make more audiences can never leave home, convenient and quick, immersive real feelings of the minority cultural items and art exhibits, to better appreciate and feel the artistic charm of the minority cultural heritage, also in the very great degree for the spreading of cultural heritage protection and has played a positive role.How to make good use of modern means of science and technology to the existing culture to carry on the protection and utilization, has become a problem urgently to be solved in each country is worth thinking and ${ }^{[6]}$.

\section{Acknowledgements}

Projects of the National Social Science Foundation of China"A Study on the Digitized Construction of the Traditional Patterns, texture and Related Cultural Relics of The unique minority Nationalities in Gansu" (Grant no.15xmz035); National Natural Science Foundation of China"Research on Key Technologies of mass video concentration based on compressed domain" (Grant no.61462057);Lanzhou science and technology development project"Unique ethnic culture information resources digitalization construction in Gansu province"(Grant no.2015-3-30).

\section{References}

[1] Gao Chengjun,Su YiXing. Unique cultural heritage protection in gansu province present situation investigation [J]. Journal of the Northwest Adult Education, 2009 (1) : 35-36.

[2] Woods Clyde.Cultural change[M]. Kunming: Yunnan Education Press, 1989-40.

[3] Wu ShanDan.Design of the Mongolian traditional digital technology research [D]. Inner Mongolia: Inner Mongolia agricultural university, 2010

[4] Li YueQiong,Fan JinSong. Based on virtual reality technology in constructing "digital temple," foshan[J]. Journal of south China normal university. 2013, (05) : P106 - P109

[5] Li XiangWei,Kang YuXiu. Key techniques of content-based video retrieval and mining [J]. Journal of software, 2014, (8) : 26 - P31

[6]Zhao Jing,Wang GaoYuan. Research on digital national costume pattern culture protection and [J]. Journal of arts education research, 2011 (12) : 21-21. 